\title{
Habilidades Sociais de Trabalhadores Com e Sem Deficiência Física ${ }^{1}$
}

\author{
Camila de Sousa Pereira ${ }^{2}$ \\ Almir Del Prette \\ Zilda Aparecida Pereira Del Prette \\ Universidade Federal de São Carlos
}

\begin{abstract}
RESUMO - Com o objetivo de comparar as habilidades sociais de trabalhadores com deficiência física (TDF) e sem deficiência física (TND) e as possíveis diferenças de sexo associadas, 27 participantes do grupo TDF e 27 do grupo TND responderam ao Inventário de Habilidades Sociais (IHS-Del-Prette) e ao Critério de Classificação Econômica Brasil. Os resultados da estatística inferencial não mostraram diferenças significativas entre os grupos e nem entre os homens TDF e TND nos escores fatoriais do IHS-Del-Prette. Houve diferença apenas no fator Autoafirmação na expressão de sentimento positivo, favorecendo as mulheres do grupo TND. São discutidas as diferenças e semelhanças entre grupos e participantes de mesmo sexo, as possíveis variáveis associadas e suas implicações para a reabilitação psicossocial e para a Educação Especial.
\end{abstract}

Palavras-chave: habilidades sociais; deficiência física; trabalhadores; trabalho.

\section{Workers Social Skills With And Without Physical Disability}

\begin{abstract}
Aiming to compare workers social skills with and without physical disabilities (TDF and TND, respectively) and possible sex-related differences, 27 TDF and 27 TND participants completed the Social Skills Inventory (IHS-Del-Prette) and the Brazil Economic Classification Criterion. The results of the inferential statistical analysis didn't show significant differences between groups and between TDF and TND males in the factorial scores of the IHS-Del-Prette. There was difference only in the factor Self-assertion in the expression of positive feeling, favoring the TND female group. Differences and similarities between groups and same sex participants, some possible related variables, and the implications for psychosocial rehabilitation and for the Special Education are discussed.
\end{abstract}

Keywords: social skills; physical disability; workers; work.

As relações interpessoais se estabelecem a partir de interações que se dão sempre sob determinadas características do ambiente sócio-cultural e são por ele influenciadas. O trabalho e as atividades ocupacionais em geral constituem contextos essencialmente interativos e, portanto, demandam habilidades de relacionamento interpessoal, também contribuindo para o desenvolvimento desse repertório. A investigação das habilidades sociais próprias desse universo está presente desde as formulações iniciais da área do Treinamento de Habilidades Sociais por Argyle (1978), considerado o pioneiro desse campo de pesquisa. Atualmente, ponderando os avanços desse campo teórico-prático, a complexidade do mundo do trabalho e a inserção do indivíduo com deficiência no trabalho formal, novas questões ganham relevância.

Segundo Del Prette, A. e Del Prette, Z. (2001), o conceito de habilidades sociais pode ser compreendido a partir da descrição de classes e subclasses de desempenhos sociais, presentes no repertório comportamental de um indivíduo,

1 Este artigo é parte modificada da Dissertação de Mestrado realizada pela primeira autora no Programa de Pós-Graduação em Educação Especial da UFSCar, sob a orientação do segundo autor. A terceira autora colaborou na elaboração desta versão. Apoio financeiro: CAPES. Os autores agradecem Andréa Regina Rosin Pinola pela leitura e sugestões no preparo deste artigo.

2 Endereço para correspondência: Universidade Federal de São Carlos, Departamento de Psicologia, Laboratório de Interação Social (LIS). Rodovia Washington Luís, Km 235, Caixa Postal: 676. São Carlos, SP. CEP13565-905.E-mail: cspereira@ufscar.br. para lidar com as situações interpessoais. Especificamente para o contexto do trabalho, esses autores propõem o conceito de habilidades sociais profissionais, definindo-as como "aquelas que atendem às diferentes demandas interpessoais do ambiente de trabalho objetivando o cumprimento de metas, a preservação do bem-estar da equipe e o respeito aos direitos de cada um" (p. 89).

Conforme a literatura especializada, as dificuldades interpessoais podem ser superadas, uma vez que as habilidades sociais são aprendidas e aprimoradas ao longo do desenvolvimento humano (Del Prette \& Del Prette, 1999; Rios, Del Prette \& Del Prette, 2002; Sánchez \& León Rubio, 2001). As experiências interpessoais e as condições ambientais influenciam a aprendizagem de habilidades sociais de diversas maneiras como, por exemplo, consequenciando ou modelando os comportamentos sociais das pessoas.

As variáveis ambientais, de acordo com Del Prette e Del Prette (2005), tanto podem promover um elaborado repertório de habilidades sociais, quanto restringi-lo, gerando déficits ou dificuldades interpessoais. Para esses autores, os fatores associados à dificuldade em apresentar um bom desempenho social são: (a) falta de conhecimento do ambiente; (b) restrições de oportunidades e modelos; (c) falhas de reforçamento; (d) ausência de feedback; (e) excesso de ansiedade interpessoal; (f) dificuldades de discriminação e processamento; e (g) problemas de comportamento internalizantes ou externalizantes. 
Esses fatores também operam sobre a aquisição e o desempenho de comportamentos socialmente habilidosos no caso das pessoas com deficiência física. A deficiência física implica no comprometimento de alguma capacidade motora e na alteração da estrutura de um ou mais segmentos do corpo humano, podendo ser uma condição congênita ou adquirida, estável ou progressiva. Geralmente, a sua ocorrência envolve parte óssea, articular, muscular ou nervosa, observando-se, nessa classificação, alterações anatômicas e fisiológicas do aparelho locomotor, com prejuízos no funcionamento do indivíduo (Hallahan \& Kauffman, 2003).

Associada a essa limitação motora, a literatura sugere problemas sociais e emocionais (e.g., autoestima baixa, imagem corporal negativa, autoaceitação reduzida, insegurança, crenças disfuncionais e isolamento social) que podem prejudicar o aprendizado das habilidades sociais, a qualidade das relações interpessoais e até mesmo as iniciativas de contatos sociais (El-Khatib, 1994; Ferreira \& Botomé, 1984; Lantican, Birdwell \& Harrell, 1994; Thomas, Bax \& Smyth, 1988). Para ilustrar, Lantican e cols. (1994) identificaram indicadores de raiva, dependência, ansiedade, autodepreciação e conflitos interpessoais em oito indivíduos com deficiência física submetidos à psicoterapia. Em outra pesquisa (El-Khatib, 1994), com 246 pessoas com deficiência física, os principais resultados revelaram dificuldades no desempenho social e na manutenção de vínculos com outras pessoas, além do relato de problemas econômicos e assistenciais, baixas perspectivas futuras e preconceito da sociedade.

Embora qualquer pessoa possa passar por conflitos interpessoais, Thomas e cols. (1988) atestaram que indivíduos com deficiência física vivenciam dificuldades mais frequentes e severas. Nesse estudo, verificou-se que $75 \%$ do grupo com deficiência física $(N=57)$ e $70 \%$ do grupo sem deficiência física $(N=62)$ enfrentavam problemas interpessoais. Todavia, $37 \%$ das pessoas com deficiência física relataram dificuldades significativamente maiores em comparação com apenas $3 \%$ do grupo sem deficiência, em situações que requeriam habilidades sociais como frequentar festas e bares, fazer amizade, oferecer ajuda ao colega de trabalho, iniciar conversação e discordar.

Essas pesquisas (El-Khatib, 1994; Lantican \& cols., 1994; Thomas \& cols., 1988) sugerem que as dificuldades enfrentadas pela pessoa com deficiência física podem tanto ser situadas no âmbito pessoal quanto ambiental. Para Del Prette e Del Prette (2005), mesmo que os indivíduos com deficiência disponham de recursos pessoais, a expressão negativa da sociedade em relação ao desviante pode constituir obstáculo à inclusão social. Considerando a importância das habilidades sociais no campo da Educação Especial, Del Prette e Del Prette (2004) sinalizam a necessidade de estudos que permitam "estabelecer parâmetros esperados" para essa população, assim como "identificar déficits e recursos de desempenho social ... que apontem para objetivos de ensino socialmente relevantes" (p. 149).

No contexto específico do trabalho, a preocupação com as consequências negativas dos problemas interpessoais tornou-se objeto de pesquisas no Brasil e em outros países, remetendo, inclusive, a questões sobre a profissionalização e a contratação de profissionais com ou sem deficiência (Dalferth, Schnappauf \& Sommerer, 1995; Del Prette, Z. \&
Del Prette, A., 2003; Pereira, Del Prette \& Del Prette, 2004; Sánchez \& León Rubio, 2001; Tanaka \& Manzini, 2005). Percebe-se que os trabalhadores com repertório de habilidades sociais mais elaborados tendem a obter êxitos nos processos seletivos para emprego e na atuação profissional, além de proporcionar para si e seu entorno bem-estar no trabalho e clima organizacional satisfatório (Câmara, Sarriera \& Pizzinato, 2004; Del Prette, A. \& Del Prette, Z., 2003; Maynard, 2003; Pereira \& Del Prette, 2007; Sarriera, Câmara \& Berlim, 2006; Wright \& Cropanzano, 2000).

Não se ignora que as dificuldades de inserção no mundo do trabalho decorrem, atualmente, de múltiplos fatores e, em particular, de fatores estruturais e conjunturais, como a redução de empregos, baixa qualificação profissional, reestruturação produtiva (Antunes, 2003; Chahad, 2003; Forrester, 1997; Lassance \& Sparta, 2003; Peres, Santos \& Carvalho, 2003). Há também os fatores culturais que remetem a características diversificadas, como é o caso do sexo. Historicamente, as condições de inserção e valorização do trabalho masculino e feminino são bastante diferenciadas, com menores oportunidades de salários e de promoção para a mulher (Giuberti \& Menezes-Filho, 2005; Nogueira, 2003). Ainda que, nas últimas décadas, essas diferenças tenham se atenuado, elas continuam existindo (Giuberti \& MenezesFilho, 2005) e, provavelmente, se manterão por muito tempo. Relacionada a essa diferença, pode-se destacar a valorização cultural da aparência física e da beleza (Câmara \& cols., 2004), que impõe maior cobrança para o sexo feminino. Reforçando as exigências para as mulheres, quanto ao desempenho social, pesquisas empíricas (Del Prette, Z. \& Del Prette, A., 2001; Del Prette \& cols., 2004; Papalia, 2003) sugerem resultados favoráveis ao adulto do sexo masculino em situações que demandam assertividade, autoexposição e controle da agressividade. Assim, pode-se facilmente supor que essas imposições culturais são ainda mais críticas para pessoas com deficiência física e, particularmente, as do sexo feminino.

Considerando, portanto: (1) a experiência negativa vivenciada pela maioria da população com deficiência física, influenciando em seu desenvolvimento socioemocional; (2) a importância da maximização do repertório de habilidades sociais como fator de inclusão no mundo do trabalho; (3) a escassez de produção científica na literatura brasileira, relacionando habilidades sociais e deficiência física, este estudo buscou: (1) analisar as habilidades sociais de trabalhadores com deficiência física em comparação com trabalhadores sem deficiência física e (2) identificar as diferenças entre mulheres com e sem deficiência física e homens com e sem deficiência física.

\section{Método}

\section{Participantes}

A amostra foi composta por 54 participantes distribuídos em dois grupos de 27 trabalhadores (14 do sexo masculino e 13 do feminino) - sendo um grupo com deficiência física (TDF) e outro sem deficiência física (TND) - ambos em- 
parelhados, conforme critérios de seleção da amostra, nas variáveis: sexo, idade, classe econômica e função. A análise estatística (Qui-quadrado e Teste-t de Student) mostrou que não houve diferenças entre os grupos nessas variáveis.

A Tabela 1 apresenta as características da amostra, subdividida pelos grupos TDF e TND.

Observa-se na Tabela 1 que a maioria dos participantes dos dois grupos tinha idade entre 18 e 37 anos, possuía o Ensino Médio Completo e era solteiro. No grupo TDF, a maioria pertencia à classe $\mathrm{C}$ e no grupo $\mathrm{TND}$, à classe $\mathrm{B} 2$.

Os participantes de ambos os grupos ocupavam os cargos de auxiliar administrativo (51,9\%), operador de caixa $(11,1 \%)$, recepcionista $(11,1 \%)$, auxiliar de almoxarifado $(7,4 \%)$, auxiliar de compras $(7,4 \%)$, operador de telemarketing $(3,7 \%)$, telefonista $(3,7 \%)$ e balconista $(3,7 \%)$. A média do tempo de serviço do grupo TDF foi um ano e sete meses $(d p=1,5)$ e do grupo TND foi seis anos e um mês $(d p=5,7)$.

Os tipos de limitações físicas foram: músculo-esquelético $(48,2 \%)$, amputação $(44,4 \%)$ e neuromotor $(7,4 \%)$. Na categoria de amputações, $29,6 \%$ era membro inferior (perna), $11,1 \%$ era membro superior (braços) e 3,7\%, membro superior e inferior.

\section{Local}

A coleta de dados ocorreu em cinco empresas, quatro privadas e uma pública, de uma cidade de aproximadamente 500 mil habitantes do estado de São Paulo. Duas delas pertenciam ao comércio, duas à prestação de serviços e uma à indústria.

\section{Instrumentos}

Inventário de Habilidades Sociais - IHS-Del-Prette (Del Prette, Z. \& Del Prette, A., 2001). Trata-se de um instrumento de autorrelato para identificação do repertório de habilidades sociais de adultos com base na estimativa do respondente sobre a frequência das suas reações em determinadas situações sociais. Possui 38 itens, caracterizando diversos contextos (público e privado) e interlocutores (autoridade, familiar, desconhecido, conhecido, amigo). Por meio de cinco alternativas em uma escala tipo Likert com valores de 0 (nunca ou raramente), 1 (com pouco frequência), 2 (com regular frequência), 3 (muito frequentemente) e 4 (sempre

Tabela 1. Características sociodemográficas da amostra, subdivididas pelos grupos.

\begin{tabular}{|c|c|c|c|c|c|}
\hline \multirow[b]{2}{*}{ Variáveis } & \multirow[b]{2}{*}{ Níveis } & \multicolumn{2}{|c|}{ Grupo TDF (N=27) } & \multicolumn{2}{|c|}{ Grupo TND (N=27) } \\
\hline & & $\begin{array}{c}\text { Frequência } \\
\text { Absoluta }\end{array}$ & $\begin{array}{c}\text { Frequência } \\
\text { Relativa }\end{array}$ & $\begin{array}{c}\text { Frequência } \\
\text { Absoluta }\end{array}$ & $\begin{array}{c}\text { Frequência } \\
\text { Relativa }\end{array}$ \\
\hline \multirow{2}{*}{ Sexo } & Feminino & 13 & 48,1 & 13 & 48,1 \\
\hline & Masculino & 14 & 51,9 & 14 & 51,9 \\
\hline \multirow{4}{*}{ Idade } & 18 a 27 anos & 13 & 48,2 & 12 & 44,5 \\
\hline & 28 a 37 anos & 10 & 37,0 & 11 & 40,7 \\
\hline & 38 a 47 anos & 4 & 14,8 & 4 & 14,8 \\
\hline & Média (DP) & \multicolumn{2}{|c|}{$29,0(7,3)$} & \multicolumn{2}{|c|}{$28,9(7,9)$} \\
\hline \multirow{3}{*}{ Escolaridade } & Médio Completo & 24 & 88,9 & 15 & 55,6 \\
\hline & $\begin{array}{l}\text { Superior } \\
\text { Incompleto }\end{array}$ & 2 & 7,4 & 8 & 29,6 \\
\hline & $\begin{array}{l}\text { Superior } \\
\text { Completo }\end{array}$ & 1 & 3,7 & 4 & 14,8 \\
\hline \multirow{5}{*}{ Classe Econômica } & A1 & - & - & 1 & 3,7 \\
\hline & A2 & - & - & 2 & 7,4 \\
\hline & B1 & 6 & 22,2 & 4 & 14,8 \\
\hline & B2 & 9 & 33,3 & 12 & 44,5 \\
\hline & $\mathrm{C}$ & 12 & 44,5 & 8 & 29,6 \\
\hline \multirow{4}{*}{ Estado Civil } & Solteiro & 19 & 70,4 & 15 & 55,6 \\
\hline & Casado & 6 & 22,2 & 12 & 44,4 \\
\hline & Divorciado & 1 & 3,7 & - & - \\
\hline & Viúvo & 1 & 3,7 & - & - \\
\hline
\end{tabular}

Nota: $\mathrm{TDF}=$ trabalhadores com deficiência física; $\mathrm{TND}=$ trabalhadores sem deficiência física; $\mathrm{dp}=\mathrm{desvio}$ padrão. 
Tabela 2. Análise descritiva e de comparação entre os grupos TDF e TND no IHS-Del-Prette.

\begin{tabular}{|c|c|c|c|c|}
\hline \multirow{2}{*}{ IHS-Del-Prette } & \multirow{2}{*}{$\begin{array}{c}\text { Grupo TDF } \\
(\mathrm{N}=27)\end{array}$} & \multirow{2}{*}{$\begin{array}{c}\text { Grupo TND } \\
(\mathrm{N}=27) \\
\text { Mediana }\end{array}$} & \multicolumn{2}{|c|}{ Mann-Whitney } \\
\hline & & & $U$ & $p$ \\
\hline Escore Total & 109,0 & 106,0 & 314,0 & 0,38 \\
\hline F1 - Enfrentamento e autoafirmação com risco & 10,6 & 12,2 & 299,0 & 0,26 \\
\hline F2 - Autoafirmação na expressão de sentimento positivo & 9,7 & 10,2 & 278,5 & 0,14 \\
\hline F3 - Conversação e desenvoltura social & 7,9 & 7,3 & 353,5 & 0,85 \\
\hline F4 - Autoexposição a desconhecidos e situações novas & 3,7 & 3,2 & 332,5 & 0,58 \\
\hline F5 - Autocontrole da agressividade & 1,3 & 1,1 & 341,0 & 0,68 \\
\hline
\end{tabular}

Nota: $\mathrm{TDF}=$ trabalhadores com deficiência física; $\mathrm{TND}=$ trabalhadores sem deficiência física.

ou quase sempre), o inventário produz um escore total e cinco fatores: F1 - Enfrentamento e autoafirmação com risco; F2 - Autoafirmação na expressão de sentimento positivo; F3 - Conversação e desenvoltura social; F4 - Autoexposição a desconhecidos e situações novas; e F5 - Autocontrole da agressividade. A validação do IHS-Del-Prette para o contexto brasileiro foi conduzida pelos autores com um grupo amostral de universitários. De acordo com vários estudos (Bandeira, Costa, Del Prette, Del Prette \& Gerk-Carneiro, 2000; Barreto, Del Prette \& Del Prette, 1998), esse instrumento apresenta qualidades psicométricas satisfatórias em termos de confiabilidade, consistência interna, estabilidade teste-reteste e validade concomitante (com o Inventário de Assertividade de Rathus). Vale destacar que o IHS-Del-Prette foi aprovado pelo Conselho Federal de Psicologia.

Critério de Classificação Econômica Brasil - CCEB (Associação Brasileira de Empresas de Pesquisa, 2003) Instrumento de domínio público que mede o poder aquisitivo do consumidor e vem sendo utilizado para classificar a população em termos econômicos. O sistema de pontuação é baseado na posse de bens de consumo duráveis, instrução do chefe da família e outros fatores, como a presença de empregados mensalistas. O CCEB divide a população brasileira em sete classes de poder aquisitivo. O percentual da população, no ano 2000, em cada classe, é o seguinte (da classe de maior poder aquisitivo para a de menor): A1 (1\%), A2 (5\%), B1 ( $9 \%)$, B2 (14\%), C (36\%), D (31\%) e E (4\%).

\section{Procedimento}

A aplicação dos instrumentos foi conduzida individualmente em uma mesma sessão. Todos os participantes receberam informações sobre a pesquisa e consentiram em colaborar assinando o Termo de Consentimento Livre e Esclarecido (estudo aprovado pelo Comitê de Ética em Pesquisa em Seres Humanos da UFSCar, parecer $\left.n^{\circ} 168 / 04\right)$. A coleta de dados foi feita pela primeira autora. Os participantes foram instruídos quanto ao preenchimento dos itens do CCEB. Na sequência, os participantes responderam ao IHS-Del-Prette, seguindo as orientações dadas conforme as normas do manual desse instrumento.

\section{Análise de dados}

O resultado do CCEB foi computado de acordo com as normas de pontuação do instrumento, obtendo-se a frequência de participantes em cada uma das classes econômicas. Os resultados do IHS-Del-Prette foram analisados considerando o escore total, os escores fatoriais e a frequência assinalada a cada um dos itens. Os itens assinalados pelos participantes na escala nunca ou raramente ou com pouca frequência foram avaliados como deficitários ou críticos em relação aos demais e, por isso, potencialmente indicadores de necessidades de intervenção. Considerando o tamanho restrito da amostra e a distribuição assimétrica dos dados, a comparação entre os grupos e entre os participantes de mesmo sexo foi feita com base em análise estatística não paramétrica (Teste MannWhitney) e dados descritivos de mediana, computados por meio do software estatístico SPSS 10.0 for Windows (Statistical Package for Social Sciences). Adotou-se o nível de significância menor ou igual a 0,05 .

\section{Resultados e Discussão}

Os resultados são apresentados a seguir sob dois tópicos: (1) Diferenças gerais entre os grupos com e sem deficiência física (TDF e TND); (2) Diferenças intergrupos para participantes de mesmo sexo. Uma discussão parcial é feita juntamente com a apresentação de cada conjunto de resultados.

\section{Diferenças gerais entre TDF e TND}

Os resultados descritivos e de comparação do escore total e dos escores fatoriais entre os grupos TDF e TND, no IHS-Del-Prette, são revelados na Tabela 2 .

Não obstante as aparentes diferenças entre os grupos TDF e TND, a análise estatística de comparação não apontou diferença significativa no escore total, tampouco nos escores dos fatores.

Em uma análise mais detalhada, a Figura 1 mostra o valor mediano de frequência de cada um dos itens do IHS-DelPrette, para os grupos TDF e TND. 
- TND $=$ TDF

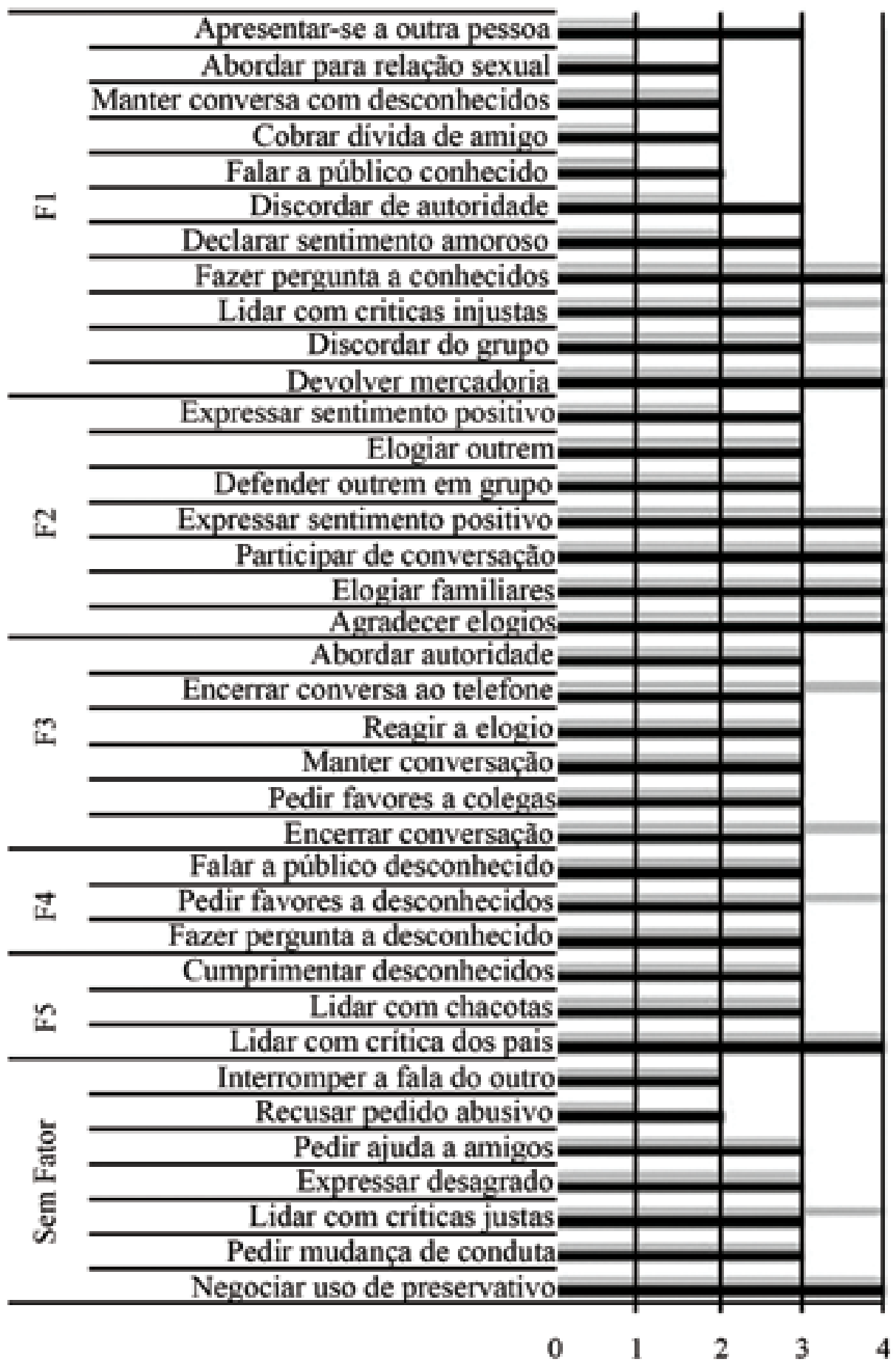

Figura 1. Mediana dos valores apontados pelos participantes nos itens de habilidades sociais do IHS-Del-Prette. Escala tipo Likert que varia de 0 (nunca ou raramente), 1 (com pouca frequência), 2 (com regular frequência), 3 (muito frequentemente) a 4 (sempre ou quase sempre). TDF=Grupo de trabalhadores com deficiência física, $\mathrm{TND}=$ Grupo de trabalhadores sem deficiência física.

Considerando os valores mais críticos em cada grupo, evidencia-se apenas o TDF, sendo que nesse grupo foram identificados quatro itens do F1 - Enfrentamento e autoafirmação com risco (Apresentar-se a outra pessoa, Abordar para relação sexual, Cobrar dívida de amigo e Falar a público conhecido) e um item não incluído nos fatores
(Recusar pedido abusivo), mas que também constitui um indicador de assertividade. Nesses itens, os participantes do grupo TDF relataram emitir com pouca frequência tais habilidades sociais.

Com esse resultado, foi possível notar que somente o grupo TDF apresentou itens com frequências inferiores 
ao esperado em habilidades sociais, em comparação com o TND, embora a estatística inferencial tenha indicado diferença significativa entre os grupos apenas no item Apresentar-se a outra pessoa $(U=209,0 ; p=0,006)$. De modo geral, esses achados são parecidos aos encontrados na pesquisa de Thomas e cols. (1988), na qual as dificuldades interpessoais em situações que exigem habilidades públicas, de amizade e de comunicação foram relatadas por jovens com e sem deficiência física, ainda que as pessoas com deficiência física tenham relatado dificuldades mais intensas nesses tipos de demandas.

É importante observar que a maior parte dos itens com déficit de desempenho do grupo TDF refere-se ao fator que avalia "a capacidade de lidar com situações interpessoais que demandam a afirmação e defesa de direitos e autoestima com risco potencial de reação indesejável por parte do interlocutor" (Del Prette, Z. \& Del Prette, A., 2001, p. 28). Com o intuito de aumentar os próprios recursos de socialização e de exercício da cidadania, muitos estudos (Chadsey \& Beyer, 2001; Dalferth \& cols., 1995; Del Prette \& Del Prette, 2004; Tanaka \& Manzini, 2005) sugerem às instituições educacionais que as habilidades sociais das pessoas com diversas deficiências - inclusive físicas - devem ser ensinadas e/ou aprimoradas. Segundo Bueno e cols. (2005) e Jaime e Carmo (2005), mesmo com os avanços de políticas públicas brasileiras no âmbito da Educação Especial, as pessoas com deficiência física ainda encontram diversos obstáculos para a participação na sociedade e no mundo do trabalho, o que torna ainda mais relevante um elaborado repertório de habilidades sociais, em especial, de enfrentamento pelo cumprimento dos seus direitos.
Diferenças intergrupos para participantes de mesmo sexo

A Tabela 3 apresenta a mediana e a comparação do escore total e dos fatores entre as mulheres do grupo TDF e TND, bem como entre os homens dos grupos TDF e TND.

Nos resultados da análise comparativa, ainda que a medida de tendência central (mediana) tenha indicado escores aparentemente superiores das mulheres do grupo TND em relação às mulheres do grupo $\mathrm{TDF}$, em todos os fatores a análise estatística inferencial apontou diferença significativa apenas no fator F2 - Autoafirmação na expressão de sentimento positivo. Entre os participantes do sexo masculino, a análise comparativa não indicou diferenças significativas entre os homens dos grupos TDF e TND no escore total, nem nos escores fatoriais.

Quanto à análise descritiva dos itens do IHS-Del-Prette, entre as mulheres do grupo TDF, as habilidades sociais mais críticas em termos de frequência de desempenho apareceram em sete dos 38 itens. A mediana apontou Falar a público conhecido, Abordar para relação sexual, Apresentar-se a outra pessoa e Falar a público desconhecido na escala nunca ou raramente emitem ou emitiriam tais habilidades. Na escala com pouca freqüência, surgiram os itens Discordar de autoridade, Cobrar dívida de amigo e Pedir ajuda a amigos. No grupo das mulheres TND, os itens deficitários foram Falar a público conhecido e Abordar para relação sexual, ambos na escala com pouca frequência de emissão dessas habilidades sociais. Na análise comparativa de todos os itens do instrumento, houve diferença significativa em Lidar com criticas dos pais $(U=45,0 ; p=0,04)$ e Interromper a fala do outro $(U=39,5 ; p=0,02)$ : as mulheres do grupo TND relataram valores superiores às do grupo TDF.

Tabela 3. Análise de medida de tendência central e comparação dos escores de habilidades sociais entre mulheres TDF e TND e entre homens TDF e TND.

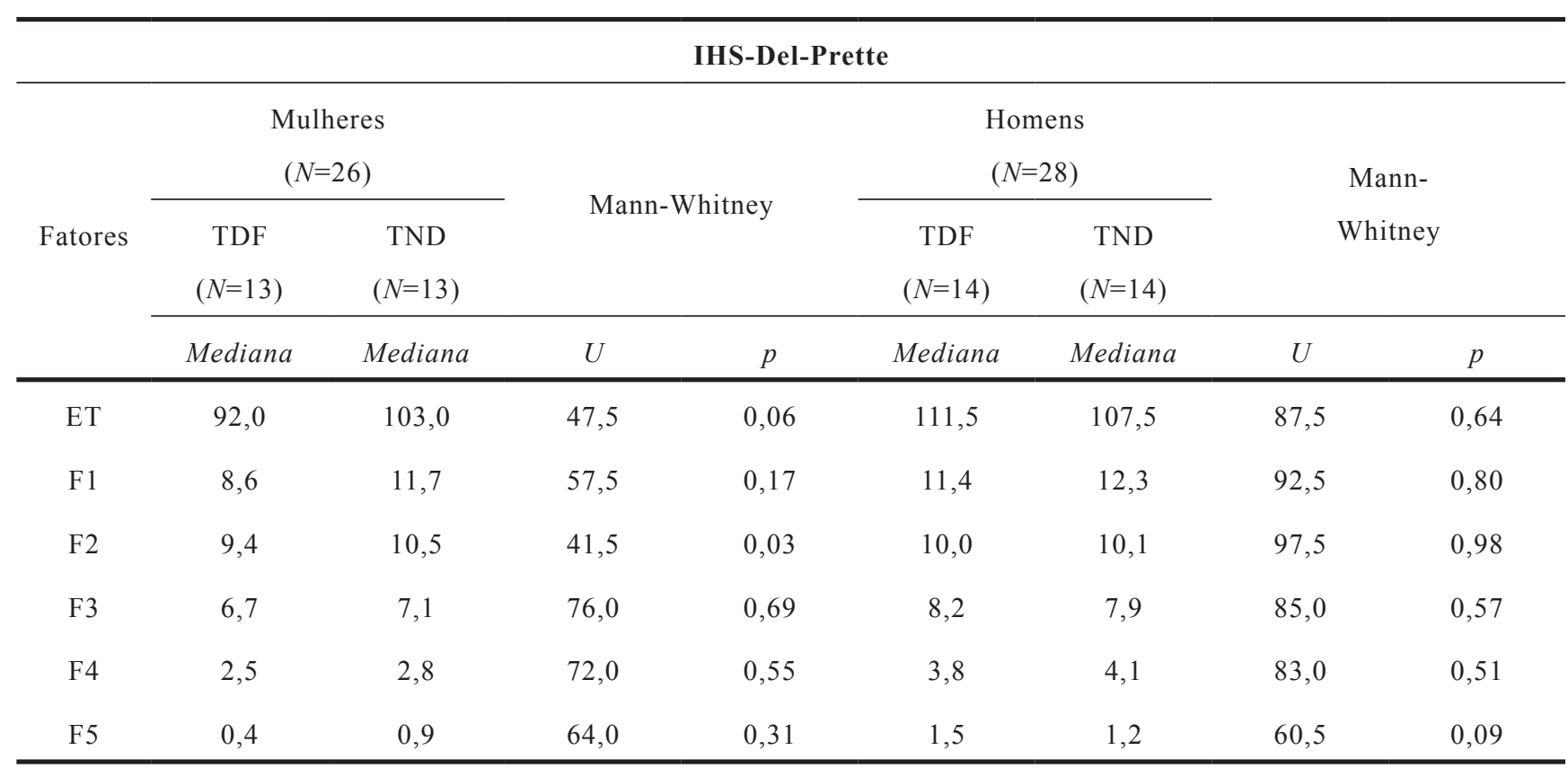

Nota: ET=Escore Total; F1=Enfrentamento e autoafirmação com risco; F2=Autoafirmação na expressão de sentimento positivo; F3=Conversação e desenvoltura social; F4=Autoexposição a desconhecidos e situações novas; F5=Autocontrole da agressividade; TDF=Trabalhadores com deficiência física; $\mathrm{TND}=$ Trabalhadores sem deficiência física. 
A análise descritiva dos itens específicos, assinalados pelos participantes do sexo masculino dos grupos TDF e TND, identificou habilidades sociais deficitárias apenas no primeiro grupo. Os homens TDF relataram emitir com pouca frequência as habilidades de Abordar para relação sexual, Apresentar-se a outra pessoa e Recusar pedido abusivo. A análise inferencial encontrou diferença significativa somente em Apresentar-se a outra pessoa ( $U=49,0$; $p=0,02$ ), no qual os homens do grupo TND relataram valores maiores aos do TDF.

Esses resultados mostram que, quando a variável deficiência física foi utilizada na comparação entre os indivíduos de mesmo sexo, as mulheres do grupo TND relataram ser mais habilidosas socialmente do que as do grupo TDF no fator que avalia a expressão de afeto positivo e nos itens referentes a lidar com críticas de familiares e interromper conversação (Del Prette, Z. \& Del Prette, A., 2001). Porém, entre os homens, as diferenças não se apresentaram significativas (com exceção do item Apresentar-se a outra pessoa). Esse resultado também é coerente com o de Thomas e cols. (1988), no qual as mulheres com deficiência física relataram maiores dificuldades em sair com uma pessoa que está interessada para relacionamento amoroso, falar sobre si mesmo e de seus próprios sentimentos quando comparadas com outras mulheres sem deficiência física.

\section{Considerações Finais}

Com os achados desta pesquisa é possível concluir que, do ponto de vista da estatística inferencial, há mais semelhanças do que diferenças entre os grupos TDF e TND em suas habilidades sociais, mas as poucas diferenças foram desfavoráveis ao TDF. Comparando os participantes de mesmo sexo com e sem deficiência física, as mulheres do grupo TDF apresentaram maiores necessidades educacionais especiais no âmbito das habilidades sociais. Além disso, a análise dos itens indicou algumas habilidades sociais relatadas com frequência inferior ao esperado, exceto entre os homens do grupo TND, das quais uma alta proporção pode ser considerada crítica para a inclusão no ambiente de trabalho, para o estabelecimento de relações interpessoais saudáveis e para o desempenho profissional.

Os resultados aqui apresentados foram baseados em instrumento de autorrelato e em apenas um indicador de desempenho (frequência). Ainda que isso não invalide os dados obtidos, um maior refinamento avaliativo seria desejável. Nesse sentido, pesquisas futuras poderiam utilizar procedimentos de avaliação multimodal das habilidades sociais (Del Prette, Casares \& Caballo, 2006), incluindo a observação direta ou até mesmo a avaliação externa por outros significantes como, por exemplo, a do supervisor, para se obter resultados mais confiáveis. Certamente, a avaliação multimodal poderia trazer informações adicionais relevantes, diminuindo os vieses na autoavaliação das habilidades sociais, complementando os resultados com a inclusão da análise dos componentes não verbais e de outros indicadores do desempenho social (como a proficiência). Não obstante a importância de tais incrementos metodológicos, os achados deste estudo já permitem subsidiar a orientação e o treinamento dos trabalhadores, tanto na perspectiva de produtividade como visando o bem-estar e a qualidade de vida no trabalho.

Outro aspecto a ser considerado é o fato do IHS-DelPrette ter seu grupo normativo ainda restrito à população universitária. Buscando diversificar o escopo no uso do instrumento, estão sendo realizadas pesquisas de ampliação da base de referência normativa em termos de escolaridade, idade e população com necessidades educacionais especiais.

Pensando em delineamentos mais complexos, novas pesquisas poderiam ser conduzidas com uma amostra maior de participantes e utilização de grupos emparelhados de trabalhadores com e sem deficiência física, bem como de desempregados com e sem deficiência física, para verificar se o trabalho pode ser uma variável que influencia no desenvolvimento do repertório de habilidades sociais. Também seria interessante investigar as diferenças no repertório de habilidades sociais entre pessoas com deficiência física congênita ou adquirida.

Considerando a inclusão do indivíduo com deficiência física no mundo do trabalho - tanto em termos de preparação profissional quanto de ajustamento social no ambiente organizacional -, os resultados deste estudo sugerem, portanto, a necessidade de pesquisas aplicadas, nas quais a variável independente seria o Treinamento de Habilidades Sociais, para verificar os possíveis efeitos desse programa sobre o desenvolvimento social e profissional de indivíduos com deficiência física. Nesse caso, os resultados do presente estudo chamam atenção especial para a necessidade de aprimoramento das habilidades de enfrentamento e de expressão de sentimento positivo, neste último, especialmente, entre as mulheres com deficiência física.

\section{Referências}

Antunes, R. (2003). Os caminhos da liofilização organizacional: as formas diferenciadas da reestruturação produtiva no Brasil. Idéias, 9/10, 13-24.

Argyle, M. (1978). Psicologia del comportamiento interpersonal. Madrid: Alianza.

Associação Brasileira de Empresas de Pesquisa (2003). Critério de Classificação Econômica Brasil. São Paulo: Autor.

Bandeira, M., Costa, M. N., Del Prette, Z. A. P., Del Prette, A., \& Gerk-Carneiro, E. (2000). Qualidades psicométricas do Inventário de Habilidades Sociais (IHS): estudo sobre a estabilidade temporal e a validade concomitante. Estudos de Psicologia, 5, 401-419.

Barreto, M. C. M., Del Prette, Z. A. P., \& Del Prette, A. (1998). Análise de itens e da estrutura fatorial de um inventário para a avaliação de repertório de habilidades sociais. Revista Brasileira de Estatística, 59(212), 7-24.

Bueno, J. G. S., Ferreira, J. R., Baptista, C. R., Oliveira, I. A., Kassar, M. M., \& Figueiredo, R. V. (2005). Políticas de educação especial no Brasil: estudo comparado das normas das unidades da federação. Revista Brasileira de Educação Especial, 11, 97-118.

Câmara, S. G., Sarriera, J. C., \& Pizzinato, A. (2004). Que portas se abrem no mercado de trabalho para os jovens em tempo de mudanças? Em J. C. Sarriera, K. B. Rocha \& A Pizzinato (Orgs.), Desafios do mundo do trabalho: orientação, inserção e mudanças (pp. 73-113). Porto Alegre: EDIPUCRS. 
Chadsey, J., \& Beyer, S. (2001). Social relationships in the workplace. Mental Retardation and Developmental, 7, 128-133.

Chahad, J. P. Z. (2003). Tendências recentes no mercado de trabalho: pesquisa de emprego e desemprego. São Paulo em Perspectiva, 17, 205-217.

Dalferth, M., Schnappauf, K., \& Sommerer, L. (1995). Vocational integration of physically handicapped persons: A regional study in the Regensburg district. Rehabilitation, 34, 91-100.

Del Prette, A., \& Del Prette, Z. A. P. (2001). Psicologia das relações interpessoais: vivências para o trabalho em grupo. Petrópolis: Vozes.

Del Prette, A., \& Del Prette, Z. A. P. (2003). No contexto da travessia para o ambiente de trabalho: treinamento de habilidades sociais com universitários. Estudos de Psicologia, 8, 413-420.

Del Prette, Z. A. P., \& Del Prette, A. (1999). Psicologia das habilidades sociais: terapia e educação. Petrópolis: Vozes.

Del Prette, Z. A. P., \& Del Prette, A. (2001). Inventário de Habilidades Sociais (IHS-Del-Prette): manual de aplicação, apuração e interpretação. São Paulo: Casa do Psicólogo.

Del Prette, Z. A. P., \& Del Prette, A. (2003). Desenvolvimento interpessoal: uma questão pendente no ensino universitário. Em E. Mercuri \& S. A. J. Polydoro (Orgs.), Estudante universitário: características e experiências de formação (pp.105-128). Taubaté: Cabral.

Del Prette, Z. A. P., \& Del Prette, A. (2004). Avaliação do repertório social de crianças com necessidades educacionais especiais. Em E. G. Mendes, M. A. Almeida \& L. C. A. Williams (Orgs.), Temas em educação especial: avanços recentes (pp. 149157). São Carlos: EDUFSCar.

Del Prette, Z. A. P., \& Del Prette, A. (2005). Psicologia das habilidades sociais na infância: teoria e prática. Petrópolis: Vozes.

Del Prette, Z. A. P., Casares, M. I. M., \& Caballo, V. E. (2006). La evaluación del repertorio de las habilidades sociales en niños. Em V. E. Caballo (Org.), Manual para la evaluación clínica de los trastornos psicológicos: trastornos de la edad adulta e informes psicológicos (pp. 373-399). Madrid: Ediciones Pirámide.

Del Prette, Z. A. P., Del Prette, A., Barreto, M. C. M., Bandeira, M., Rios-Saldaña, M. R., Ulian, A. L. A. O., Gerk-Carneiro, E., Falcone, E. M. O., \& Villa, M. B. (2004). Habilidades sociais de estudantes de Psicología: um estudo multicêntrico. Psicologia: Reflexão e Crítica, 17, 341-350.

El-Khatib, U. (1994). As dificuldades das pessoas portadoras de deficiência física: quais são e onde estão? Dissertação de Mestrado, Universidade de São Paulo. São Paulo.

Ferreira, M. R., \& Botomé, S. P. (1984). Deficiência física e inserção social: a formação dos recursos humanos. Caxias do Sul: EDUSC.

Forrester, V. (1997). O horror econômico. São Paulo: UNESP.

Giuberti, A. C., \& Menezes-Filho, N. (2005). Discriminação de rendimentos por gênero: uma comparação entre o Brasil e os Estados Unidos. Economia Aplicada, 9, 369-383.

Hallahan, D. P., \& Kauffman, J. M. (2003). Exceptional learners: Introduction to special education (pp. 431-451). New York: Allyn and Bacon.

Jaime, L. R., \& Carmo, J. C. (2005). A inserção da pessoa com deficiência no mundo do trabalho: o resgate de um direito de cidadania. São Paulo: Editora dos Autores.
Lantican, L. S., Birdwell, C. N., \& Harrell, R. T. (1994). Physically handicapped individuals in psychotherapy: Some empirical data. Issues in Mental Health Nursing, 15, 73-84.

Lassance, M. C., \& Sparta, M. (2003). A orientação profissional e as transformações do mundo do trabalho. Revista Brasileira de Orientação Profissional, 4(1/2), 13-19.

Maynard, M. L. (2003). Emotional intelligence and perceived employability for internship curriculum. Psychological Reports, 93, 791-792.

Nogueira, C. M. (2003). A feminização no mundo do trabalho: entre a emancipação e a precarização. Idéias, 9/10, 247-284.

Papalia, D. E. (2003). Human development. New York: McGraw-Hill.

Pereira, C. S., \& Del Prette, A. (2007). Vendedor com paralisia cerebral bem-sucedido: análise de um filme na perspectiva das habilidades sociais. Revista Brasileira de Orientação Profissional, 8(2), 87-91.

Pereira, C. S., Del Prette, A., \& Del Prette, Z. A. P. (2004). A importância das habilidades sociais na função do técnico em segurança do trabalho. Argumento, 6(12), 103-113.

Peres, R. S., Santos, M. A., \& Carvalho, A. M. R. (2003). Precarização das relações de trabalho, desemprego e desigualdade social: desafios para a Psicologia na economia globalizada. Em L. L. Melo-Silva, M. A. Santos \& M. C. Avi (Orgs.), Arquitetura de uma ocupação - Orientação profissional: teoria e prática, Vol 1 (pp. 173-185). São Paulo: Vetor.

Rios, M. R. S., Del Prette, A., \& Del Prette, Z. A. P. (2002). A importância da Teoria da Aprendizagem Social na constituição da área do treinamento de habilidades sociais. Em H. J. Guilhardi, M. B. B. P. Madi, P. P. Queiroz \& M. C. Scoz (Orgs.), Sobre comportamento e cognição: contribuições para a construção da Teoria do Comportamento, Vol. 9 (pp. 269-283). Santo André: ESETec.

Sánchez, M. M., \& León Rubio, J. M. (2001). Entrenamiento en habilidades sociales: un método de enseñanza-aprendizaje para desarrollar las habilidades de comunicación interpersonal en el área de enfermería. Psicothema, 13, 247-251.

Sarriera, J. C., Câmara, S. G., \& Berlim, C. S. (2006). Formação e orientação ocupacional: manual para jovens à procura de emprego. Porto Alegre: Sulina.

Tanaka, E. D. O., \& Manzini, E. J. (2005). O que os empregadores pensam sobre o trabalho da pessoa com deficiência? Revista Brasileira de Educação Especial, 11, 273-294.

Thomas, A. P., Bax, M. C., \& Smyth, D. P. (1988). The social skill difficulties of young adults with physical disabilities. Child: Care, Health and Development, 14, 255-264.

Wright, T. A., \& Cropanzano, R. (2000). Psychological wellbeing and job satisfaction as predictors of job performance. Journal of Occupational Health Psychology, 5, 84-94. 